\title{
Multiple nocardial abscesses of the brainstem and spinal cord diagnosed after an open biopsy through a cervical partial central corpectomy: case report
}

\author{
Ian Peeters, MD, ${ }^{1}$ Jan W. Casselman, MD, PhD, ${ }^{2}$ Stefaan J. Vandecasteele, MD, PhD, ${ }^{3}$ \\ Alexander Janssen, MD, ${ }^{4}$ Bart Regaert, MD, ${ }^{4}$ Nikolaas Vantomme, MD, ${ }^{4}$ and \\ Ludo J. Vanopdenbosch, MD'1
} Departments of ${ }^{1}$ Neurology, ${ }^{2}$ Radiology, ${ }^{3}$ Nephrology and Infectious Diseases, and ${ }^{4}$ Neurosurgery, Hospital AZ Sint-Jan,
Brugge, Belgium

\begin{abstract}
Nocardiosis of the central nervous system is a challenging and difficult diagnosis for the clinician. The combination of infections of the brain and spinal cord is even more rare. The authors report on a patient with multiple lesions in the brainstem and cervical spinal cord. This 81 -year-old immunocompetent woman presented with symptoms of progressive walking difficulty and ataxia. The results of an extensive workup with laboratory investigation, MRI, lumbar puncture, positron emission tomography (PET), and bone marrow biopsy remained inconclusive. Only after an open biopsy of a cervical lesion by an anterior approach through a partial central corpectomy of the cervical spine, was the diagnosis of nocardiosis made, allowing for specific antibiotic treatment.
\end{abstract}

http://thejns.org/doi/abs/10.3171/2014.12.SPINE14755

KEY WORDS nocardiosis; brainstem; spinal cord; biopsy; partial corpectomy; infection; cervical spine

$N$ OCARDIA is a gram-positive bacterium that usually manifests as an opportunistic infection in immunocompromised hosts. Although nocardiosis most commonly presents as a pulmonary infection, extrapulmonary localizations exist, and the central nervous system can be considered one of the primary sites of infection. Brain abscesses and spinal cord abscesses are usually described as separate and rare entities. Infection of both the brain and the spinal cord is rare, and a PubMed search revealed only 3 other cases. ${ }^{4,9,19}$ We present a challenging case in which the diagnosis of nocardiosis could only be made after an open biopsy of the cervical spine by an anterior approach through a partial central corpectomy.

\section{Case Report}

History and Clinical Examination

An 81-year-old woman presented with walking difficulty and gait instability. She had a hoarse voice and difficulty swallowing. Clinical examination showed hypermetric saccadic eye movements toward the right side. There was a subtle drift of the right arm when holding both arms upwards. In the right leg there was ataxia and loss of proprioception. When standing with the eyes closed, she showed a tendency to fall toward the right side.

\section{Investigation and Initial Treatment}

Initial MRI showed a hyperintense lesion in the brainstem starting from the cerebellar peduncle and extending to the lower endplate of $\mathrm{C}-2$. Images obtained after administration of gadolinium contrast medium showed 4 separate ring-enhancing lesions. Analysis of CSF obtained by lumbar puncture showed 94 white blood cells per microliter (38\% neutrophils, $48 \%$ lymphocytes). Empiric antibiotic therapy (ceftriaxone and ampicillin) was initiated, along with acyclovir and methylprednisolone treatment. Over the next day the patient developed general ataxia, global aphasia, and paresis of the right arm. Acyclovir treatment was discontinued 4 days after it was initiated because the polymerase chain reaction (PCR) results were negative for herpes and varicella viruses. After 2 weeks of ceftriaxone and 4 weeks of ampicillin treatment for a

ABBREVIATION PCR = polymerase chain reaction.

SUBMITTED July 29, 2014. ACCEPTED December 31, 2014.

INCLUDE WHEN CITING Published online June 19, 2015; DOI: 10.3171/2014.12.SPINE14755.

DISCLOSURE The authors report no conflict of interest concerning the materials or methods used in this study or the findings specified in this paper. 
possible Listeria infection, there was a good radiographic response on MRI but no clinical improvement. Terminating the antibiotic treatment resulted in a rapid clinical deterioration, with paralysis of the lower limbs and incontinence. Two days after terminating the antibiotic treatment a third MRI study was performed, which revealed a new ring-enhancing lesion in the cervical medulla at the level of C4-5 and demonstrated that the old lesions were still visible. A fourth MRI study performed 4 days after termination of antibiotic treatment showed further growth of this lesion from the first toward the sixth cervical vertebral level (Fig. 1).

During this time, the results of extensive laboratory investigation of blood, lumbar CSF, and stool samples remained negative for Listeria, toxoplasmosis, strongyloides, Taenia, Echinococcus species, JC virus, Aspergillus, Toxocara, Treponema pallidum, Brucella, Bartonella henselae, Q fever, and even NMO antibodies. The results of a bone marrow biopsy, performed to exclude lymphoma, were also negative. A PET scan showed nodules in the supraclavicular region and the hepatic curve of the colon. A biopsy from the supraclavicular nodule showed benign lymphoid tissue, while a biopsy of the colon (performed via colonoscopy) showed only a tubulovillous adenoma with low-grade dysplasia.

\section{Operation and Postoperative Course}

Eventually, because of the patient's clinical deterioration, explained by the new anterior ring-enhancing lesion, we decided an open biopsy was necessary. The surgical strategy was the result of several concerns. Most importantly, addressing the biggest lesion at the level of C-4 seemed logical, because of the compressive effect the lesion exerted on the medulla. We planned an evacuating puncture of the presumed necrotic core of the abscess with additional biopsy of the wall of the abscess cavity. Since the lesion was located in front of the spinal cord, an anterior approach would eliminate the need to transgress the cord and risk additional functional damage. Deciding upon the anterior approach, it was felt that an adequate exposure of the dura mater and the spinal cord was necessary to be able to access and decompress the lesion, perform hemostasis, and close the dura in a controlled manner. Although single-level discectomies and midline trough corpectomies have both been documented for the evacuation of extensive epidural abscesses without the need for additional instrumented fusion, ${ }^{3,14}$ in this case, the need to open the dura, as well as the need for instrumented fusion, warranted a wider corridor. Performing an instrumented fusion in an infected environment has been reported in previous articles. ${ }^{15,16}$ The neurosurgeon's (B.R.) comfort with and confidence in the anterior technique, in contrast to a lateral approach for a more laterally situated lesion, for example, were also taken into account. The level of incision was indicated by intraoperative fluoroscopy. A standard anterior cervical approach was used to expose the ventral wall of the vertebral bodies of C-4 and C-5. Using the microscope, corpectomies of these vertebrae were performed, sparing the uncovertebral joints to maintain maximal stability. The posterior longitudinal ligament at C4-5 was carefully removed to adequately expose the dura. The dura was incised longitudinally, and dural tenting sutures
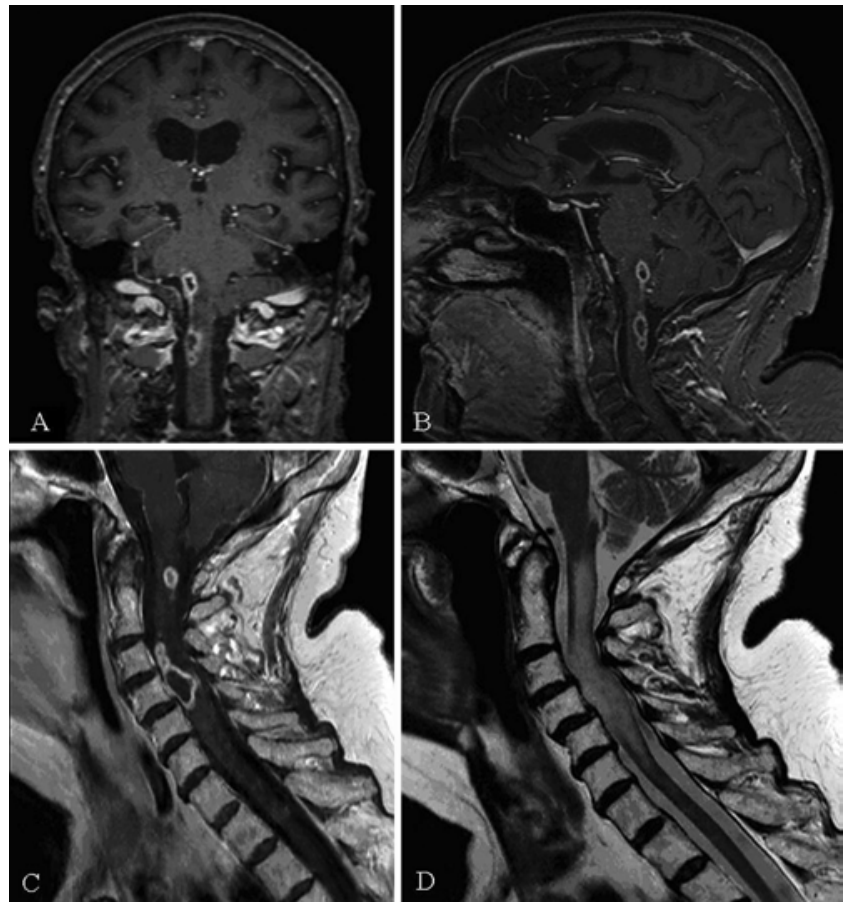

FIG. 1. A and B: Coronal and sagittal Gd-enhanced T1-weighted MR images obtained at admission showing ring-enhancing lesions in the brainstem. C and D: Sagittal Gd-enhanced T1-weighted MR image (C) and T2-weighted turbo spin echo image (D) showing a new cervical ring-enhancing lesion and hyperintense spinal cord expansion after termination of antibiotic therapy (2 weeks of ceftriaxone and 1 month of ampicillin).

were placed. At this stage, the intramedullary nature of the lesion was confirmed. The cord had a localized yellowish aspect. A small piece of the evidently diseased area was biopsied and a viscous material extruded spontaneously and was harvested for both anatomopathological examination and cultures. After confirmation of adequate hemostasis, the dura mater was closed with a running stitch. The stitch was covered by a thin layer of Tissucol (Baxter), a single layer of Bicol (DePuy Synthes, Codman Neuro), followed by one more thin layers of Tissucol. A stackable cage filled with Nanostim synthetic bone paste (Medtronic) was introduced, and an anterior cervical plate was screwed onto the bodies of C-3 and C-6 for increased stabilization. A drain was left in the prevertebral space under suction, and the wound was closed in a standard fashion (Fig. 2).

\section{Pathological Findings}

Anatomopathological examination showed inflammatory infiltrate and purulence surrounded by necrotic debris. DNA extraction and PCR for Nocardia species was performed in the national reference laboratory for nocardiosis in Lyon. PCR was clearly positive for Nocardia.

\section{Postoperative Course}

Treatment with trimethoprim/sulfamethoxazole was initiated, resulting in a good radiological and clinical response. After 2 months of intensive rehabilitation, the patient was able to leave the hospital in stable condition. She spent another month in a rehabilitation center before going home. Swallowing liquids initially remained difficult, and 

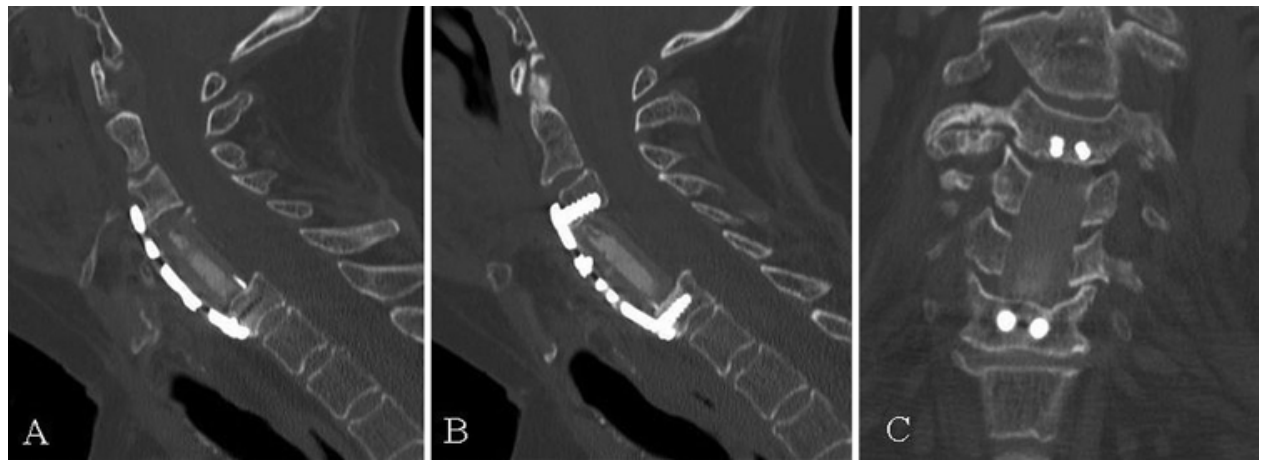

FIG. 2. Postoperative sagittal (A), parasagittal (B), and coronal (C) CT reconstructions showing a stackable cage between C-3 and C-6 filled with Nanostim synthetic bone paste and a cervical plate for increased stabilization.

a modified diet was needed the 1st month after surgery. At the time of discharge, the patient had fully recovered her swallowing function. At 6 months' follow-up the patient had minor muscle weakness on the right side and could walk independently for some distance with minimal aid. Antibiotic treatment will be continued for at least 1 year, until lesions are no longer visible on neuroimaging (Fig. 3).

\section{Discussion}

The genus Nocardia has been described as "a ubiquitous group of environmental bacteria that usually manifest as an opportunistic infection in immunocompromised hosts." ${ }^{20}$ Nocardia was first identified by Edmond Nocard in 1888. ${ }^{22}$ These organisms may be found in soil, decomposing vegetation, and other organic matter; they can also be found in fresh and salt water. ${ }^{20}$ Nocardia asteroides complex is the subgroup that causes the most clinical human nocardial infections. Nocardia is a gram-positive, partially acid-fast, catalase- and urease-positive bacterium that grows aerobically. ${ }^{22}$ Under direct microscopy, Nocardia appears as a filamentous bacterium with hyphae-like branching. ${ }^{20}$

Infection with Nocardia (nocardiosis) is usually acquired by inhalation, and therefore the most common clinical presentation is pulmonary nocardiosis. Extrapulmonary localization occurs through hematogenous dissemination or contiguous spread. The central nervous system is the most common extrapulmonary localization and can be considered as a primary site of nocardiosis. ${ }^{2}$ Supratentorial localization has been reported to be more frequent than infratentorial localization; lesions are single in $54 \%$ and multiple in $38 \%$ of cases. ${ }^{11}$ Nocardia brain abscess carries a high mortality rate-up to $34 \%$, which is considered the highest among brain abscesses caused by microorganisms. ${ }^{22}$ Solitary involvement of the spinal cord, on the other hand, is very rare, and only very few cases have been reported., ${ }^{1,6-8,12,13,21}$ Spinal abscesses are more frequently found in the lower spinal levels. Only 2 other cases involved a cervical abscess localization, and in both cases, the patients died quickly after diagnosis. ${ }^{4,17}$ The combination of brain and spinal cord abscesses is even more rare. In a review of the literature on PubMed, we found only 3 other cases of a nocardial abscess in both the brain and the spinal cord.,9,19
Different treatment options for nocardial CNS abscesses include antimicrobial therapy alone, freehand or stereotactic aspiration/biopsy, and craniotomy plus enucleation, in addition to antibiotic therapy. ${ }^{5,11,18}$ Different treatment

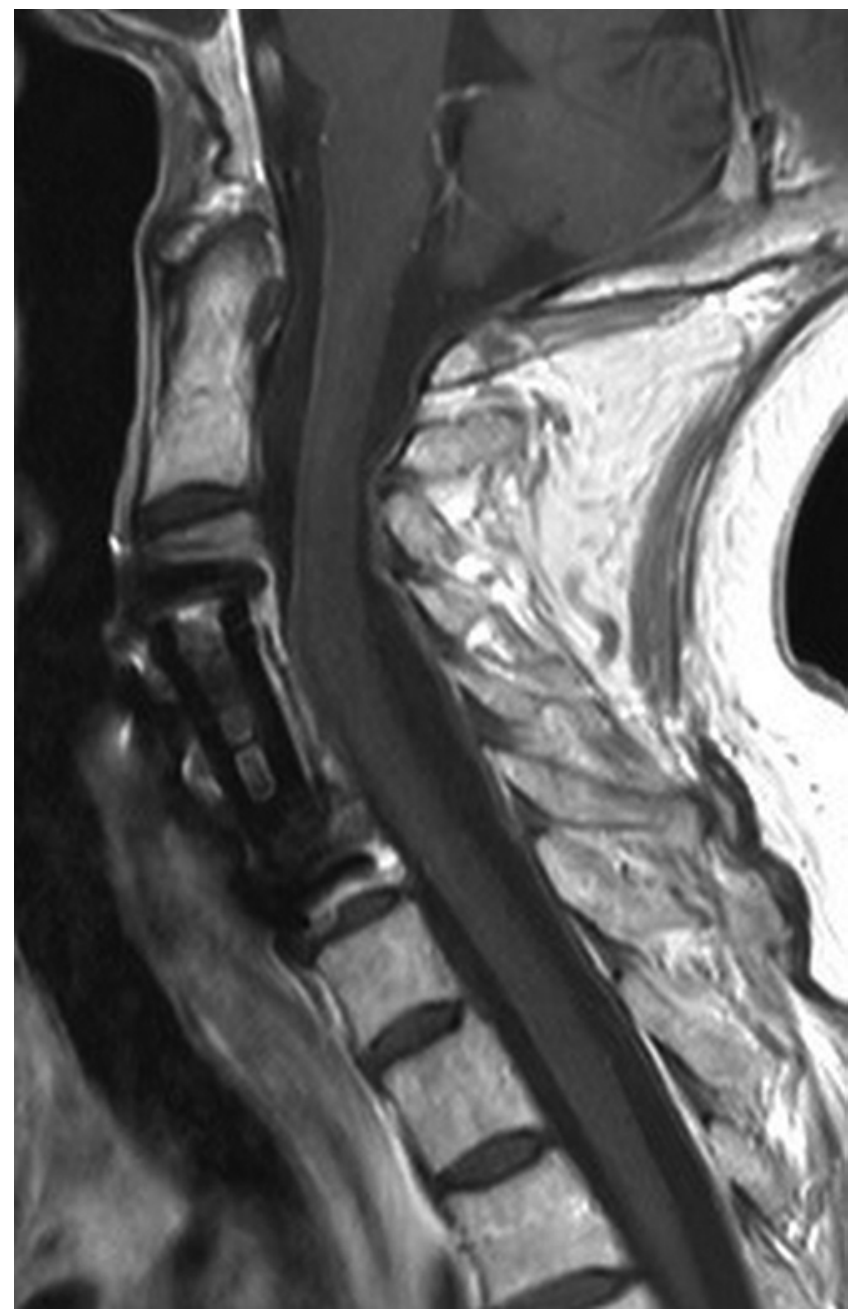

FIG. 3. Sagittal Gd-enhanced T1-weighted MR image obtained after 6 months of treatment with trimethoprim/sulfamethoxazole, showing resolution of the ring-enhancing lesions in the brainstem and cervical medulla. 
strategies have been recommended. Mamelak et al. recommend an initial trial of antibiotic therapy if an extraneural nocardial infection is documented in a clinically stable or an immunocompetent patient with a brain abscess smaller than $2 \mathrm{~cm}$ in maximum diameter. ${ }^{11}$ If the patient's condition deteriorates or if the abscess does not decrease in 4 weeks, aspiration of the lesion should be performed to confirm the diagnosis and to decompress the lesion. All abscesses larger than $2.5 \mathrm{~cm}$ in diameter should be aspirated regardless of the immune status of the patient. Serial CT or MRI should be done every 2 weeks and imaging studies should also be performed after any clinical deterioration. If the abscess enlarges after 2 weeks of treatment or remains unchanged after any clinical deterioration, craniotomy should be performed to excise the abscess. ${ }^{11}$ Lee et al. and Zakaria et al. recommend a less-aggressive approach with repeated aspirations if necessary in addition to antibiotic treatment. ${ }^{10,22}$ Concerning spinal cord abscesses, there are no clear recommendations. Based on the reported cases, it appears that laminectomy is usually performed, if feasible, to aspirate or enucleate the abscess. In the presented case, no lesion was initially accessible for aspiration or enucleation. Only when a lesion in the cervical spine was accessible was it possible to perform a biopsy.

The antibiotic agents of choice for the treatment of $\mathrm{No}$ cardia are sulfonamides. Trimethoprim/sulfamethoxazole is the most frequently used preparation, although the benefit of the trimethoprim component is unclear. Trimethoprim/sulfamethoxazole has good antimicrobial activity against most species of Nocardia and it has good penetrance through the blood-brain barrier, which is important for cerebral abscesses. Divided doses of 5 to $10 \mathrm{mg} / \mathrm{kg}$ per day of the trimethoprim component (or 25 to $50 \mathrm{mg} / \mathrm{kg}$ per day of sulfamethoxazole) are recommended to produce sulfonamide serum concentrations between 100 and 150 $\mu \mathrm{g} / \mathrm{ml}$. Other active agents include amikacin, imipenem, meropenem, ceftriaxone, cefotaxime, minocycline, moxifloxacin, levofloxacin, linezolid, tigecycline, and amoxicillin/clavulanic acid. Immunocompetent patients with pulmonary or multifocal (non-CNS) nocardiosis should be treated for at least 6 months. In immunocompromised patients or patients with CNS disease, antimicrobial treatment should be continued for at least 12 months, and clinical and radiographic monitoring should be performed frequently. ${ }^{20}$

\section{References}

1. Atalay B, Azap O, Cekinmez M, Caner H, Haberal M: Nocardial epidural abscess of the thoracic spinal cord and review of the literature. J Infect Chemother 11:169-171, 2005

2. Beaman BL, Beaman L: Nocardia species: host-parasite relationships. Clin Microbiol Rev 7:213-264, 1994

3. Deshmukh VR: Midline trough corpectomies for the evacuation of an extensive ventral cervical and upper thoracic spinal epidural abscess. J Neurosurg Spine 13:229-233, 2010

4. Durmaz R, Atasoy MA, Durmaz G, Adapinar B, Arslantaş A, Aydinli A, et al: Multiple nocardial abscesses of cerebrum, cerebellum and spinal cord, causing quadriplegia. Clin Neurol Neurosurg 103:59-62, 2001

5. Hall WA, Martinez AJ, Dummer JS, Lunsford LD: Nocardial brain abscess: diagnostic and therapeutic use of stereotactic aspiration. Surg Neurol 28:114-118, 1987
6. Hiller R, Singh H, Crone M: Left leg paralysis in a renal transplant. Am J Kidney Dis 33:E4, 1999

7. Hong SB, Han K, Son BR, Shin KS, Rim BC: First case of Nocardia nova spinal abscess in an immunocompetent patient. Braz J Infect Dis 16:196-199, 2012

8. Hussain N, Srinivasan A, Paquette S, Agbi C, Lum C: Neuroimaging highlight. MRI of spinal nocardial abscess. Can J Neurol Sci 31:404-405, 2004

9. Kampfl A, Auckenthaler A, Berek K, Birbamer G, Haring HP, Luef G, et al: [Cerebral and spinal nocardia infection. A case report.] Wien Klin Wochenschr 104:174-177, 1992 (Ger)

10. Lee GY, Daniel RT, Brophy BP, Reilly PL: Surgical treatment of nocardial brain abscesses. Neurosurgery 51:668672,2002

11. Mamelak AN, Obana WG, Flaherty JF, Rosenblum ML: Nocardial brain abscess: treatment strategies and factors influencing outcome. Neurosurgery 35:622-631, 1994

12. Mehta RS, Jain D, Chitnis DS: Nocardial abscess of spinal cord. Neurol India 47:243-244, 1999

13. Mukunda BN, Shekar R, Bass S: Solitary spinal intramedullary abscess caused by Nocardia asteroides. South Med J 92:1223-1224, 1999

14. Muzii VF, Mariottini A, Zalaffi A, Carangelo BR, Palma L: Cervical spine epidural abscess: experience with microsurgical treatment in eight cases. J Neurosurg Spine 5:392-397, 2006

15. Ogden AT, Kaiser MG: Single-stage debridement and instrumentation for pyogenic spinal infections. Neurosurg Focus 17(6):E5, 2004

16. Przybylski GJ, Sharan AD: Single-stage autogenous bone grafting and internal fixation in the surgical management of pyogenic discitis and vertebral osteomyelitis. J Neurosurg 94 (1 Suppl):1-7, 2001

17. Samkoff LM, Monajati A, Shapiro JL: Teaching NeuroImage: nocardial intramedullary spinal cord abscess. Neurology 71:e5, 2008

18. Weiss MH, Jane JA: Nocardia asteroides brain abscess successfully treated by enucleation. Case report. J Neurosurg 30:83-86, 1969

19. Welsh JD, Rhoades ER, Jaques W: Disseminated nocardiosis involving spinal cord. Case report. Arch Intern Med 108:73-79, 1961

20. Wilson JW: Nocardiosis: updates and clinical overview. Mayo Clin Proc 87:403-407, 2012

21. Yenrudi S, Shuangshoti S, Pupaibul K: Nocardiosis: report of 2 cases with review of literature in Thailand. J Med Assoc Thai 74:47-54, 1991

22. Zakaria A, Elwatidy S, Elgamal E: Nocardia brain abscess: severe CNS infection that needs aggressive management; case report. Acta Neurochir (Wien) 150:1097-1101, 2008

\section{Author Contributions \\ Conception and design: Peeters, Vanopdenbosch. Acquisition of data: Peeters, Janssen, Regaert, Vantomme. Analysis and inter- pretation of data: Peeters, Casselman, Vandecasteele, Regaert, Vantomme. Drafting the article: Peeters, Vanopdenbosch. Critically revising the article: Peeters, Casselman, Regaert, Vanopdenbosch. Reviewed submitted version of manuscript: Peeters, Vanopdenbosch. Approved the final version of the man- uscript on behalf of all authors: Peeters.}

\section{Correspondence}

Ian Peeters, Neurology-Neurosurgery, AZ Sint-Jan BruggeOostende, Ruddershove 10, Brugge B-8000, Belgium. email: ian. peeters@ugent.be. 